\title{
The Use of Intensity Modulated Radiotherapy as a Mean of Reducing Dose to Bone Marrow for Patients with Cancer Cervix Treated at Nci, Cairo, Egypt. Dosimetric Study
}

\author{
Mohamed Mahmoud1, Mahmoud Shoshaa ${ }^{2}$, Maha Hassan $^{3}$, Shaimaa Abdelgelil', Sandy Mohamed ${ }^{1}$ \\ ${ }^{1}$ Radiation Oncology Department, National Cancer Institute, Cairo University, Egypt \\ ${ }^{2}$ Radiation Physics Unit, Shefaa Alorman Oncology Hospital, Egypt \\ ${ }^{3}$ Radiation Physics Unit, National Cancer Institute, Cairo University, Egypt \\ ${ }^{4}$ Epidemiology and Cancer biostatistics Department, National Cancer Institute (NCI), Cairo University, Egypt \\ Email: m_mahmoud1973@hotmail.com
}

How to cite this paper: Mahmoud, M., Shosha, M., Hassan, M., Abdelgelil, S. and Mohamed, S. (2017) The Use of Intensity Modulated Radiotherapy as a Mean of Reducing Dose to Bone Marrow for Patients with Cancer Cervix Treated at Nci, Cairo, Egypt. Dosimetric Study. Journal of Cancer Therapy, 8, 891-901.

https://doi.org/10.4236/jct.2017.810078

Received: September 11, 2017

Accepted: October 28, 2017

Published: October 31, 2017

Copyright $\odot 2017$ by authors and Scientific Research Publishing Inc. This work is licensed under the Creative Commons Attribution International License (CC BY 4.0).

http://creativecommons.org/licenses/by/4.0/

\section{Abstract}

Purpose: To test the use of Intensity Modulated Radiotherapy (IMRT) to spare the bone marrow (BM) in patients treated with cancer cervix through using the bone marrow an organ at risk. Patients and methods: Thirteen patients with stage (IB2-IIIB) intact cervix cancer were included; C-T simulation was done with contrast and full bladder with slice thickness $2.5 \mathrm{~mm}$. Clinical Target Volume (CTV) included the cervix, uterus, upper half of the vagina, parametrium and regional lymph nodes. The CTV was expanded by $1 \mathrm{~cm}$ to form the Planning Target Volume (PTV). The organ at risk (OAR) included the bladder and rectum, the external contour of the pelvic bones to define the Pelvic Bone Marrow (PBM). Four plans were done for every patient with anteroposterior-posteroanterior (AP/PA), three dimensional conformal radiotherapy (3DCRT), IMRT, bone marrow-sparing intensity-modulated pelvic radiotherapy (BMS-IMRT). Results: BMS-IMRT reduced the V20, V30, V40 and V45 of the BM in comparison to 3DCRT and IMRT plans. Reduction in V20 with BMS-IMRT plan compared to 3DCRT ( $p<0.03)$. The PBM volume receiving 5, 10 and 20 Gy was lower AP/PA than BMS-IMRT $(p<0.01, p<$ 0.001 and $p<0.04$ respectively). The volumes of the rectum and bladder receiving dose of 40 and 45 Gy were lower in BMS-IMRT plan compared to AP/PA and 3DCRT plans with $p=0.01$ for both of them. PTV cover was better in the BMS-IMRT and IMRT plans. Conclusion: BMS-IMRT decreased the irradiated BM volume compared to other techniques. Thus using BMS-IMRT is recommended to decrease hematological toxicity and avoid 
treatment interruption.

\section{Keywords}

IMRT, BMS-IMRT, OAR, PBM

\section{Introduction}

Concurrent chemo-radiotherapy is the standard treatment practice for locally advanced cancer cervix. Randomized trials have found that chemoradiation therapy improves tumor control compared with radiation therapy (RT) alone [1] [2] also it is more effective in improving survival than radiotherapy alone.

On the other hand, hematologic toxicity from the combined treatment, results in delaying chemotherapy courses, which affect the treatment outcome [3]. Thus, it is important to decrease the incidence of acute hematologic toxicity so as to tolerate the treatment.

The lumbar, sacrum, iliac crest, ischium, pubis, and proximal femur, produces about $50 \%$ of the bone marrow [4] and these regions are exposed to various dose of radiation throughout the course of external beam radiotherapy.

Together radiation and chemotherapy are myelosuppressive, however the degree and mechanism by which radiation causes haematologic toxicity with chemotherapy are still unknown. Radiation leads to apoptosis of bone marrow (BM) stem cells and stromal damage, leading to myelosuppression of bone marrow [5].

Studies showed that the degree of bone marrow injury caused by radiation is dependent on volume of the bone marrow irradiated as well as the dose received by the bone marrow [6] [7]. Pelvic irradiation involves large volumes of active BM.

In this study, our aim is to make the BM as organ at risk in the so as to reduce the irradiated bone marrow volume in cases treated for cancer cervix.

\section{Patients and Methods}

\subsection{Patients}

Thirteen cervical cancer patients who were treated with concurrent chemotherapy-radiotherapy were included in the study. The mean and median age was 53.5 and 52 respectively. All patients were with intact cervix, with FIGO (International Federation of Gynecology and Obstetrics) stage IB2 in 2, IIB in 8, IIIA in 2 and IIIB IN 1.

Patients with early stages, stage IVA and IVB and postoperative cases were excluded from the study.

\subsection{Simulation}

This is a dosimetric study where C-T simulation was done for thirteen patients with cervix cancer with contrast and full bladder using GE-CT simulator with a 
slice thickness of $2.5 \mathrm{~mm}$. The clinical target volume (CTV) included the upper one-half of the vagina, both parametria, whole uterus, uterine cervix, presacral area, and draining lymph nodes (lower common, internal, and external iliac lymph nodes). A margin of One $\mathrm{cm}$ was added around the CTV to form the PTV. The organ at risk (OAR) included the bladder, rectum, pelvic bones marrow (PBM) including the lumbosacral BM (LSBM), iliac BM (IBM), and pubis.

Three-dimensional conformal (4-field box technique) and Anterior/Posterior (AP/PA) is planned on XIO CMS (v.5.1). Also, equally spaced nine coplanar fields IMRT (IMRT plan without BMS constraint).and BMS-IMRT is planned on Monaco (v.5.1) treatment planning system. The prescribed dose was $45 \mathrm{~Gy}$ in 25 fractions.

All plans were normalized to cover $98 \%$ of the PTV. All treatment plans are evaluated using isodose line distribution and dose-volume histogram (DVH). The PBM total (which include iliac, pubis and Lumbosacral) and OAR volumes irradiated at different dose 5, 10, 20, 30, 40 and 45 Gy was compared. The analysis was performed as mean $\pm \mathrm{SD}$ using Origin 6. $P$ value $<0.05$ is significant.

\section{Results}

The dose distribution obtained for IMRT, BMS-IMRT, AP/PA and 3DCRT techniques are shown in Figure 1. There were a slightly minor dosimetric differences observed between the four plans in PTV DVHs for all patients individually.

Figure 1, showed that BMS-IMRT does not only reduced the dose to the surrounding risk organs but also limited the dose to the PTV compared with
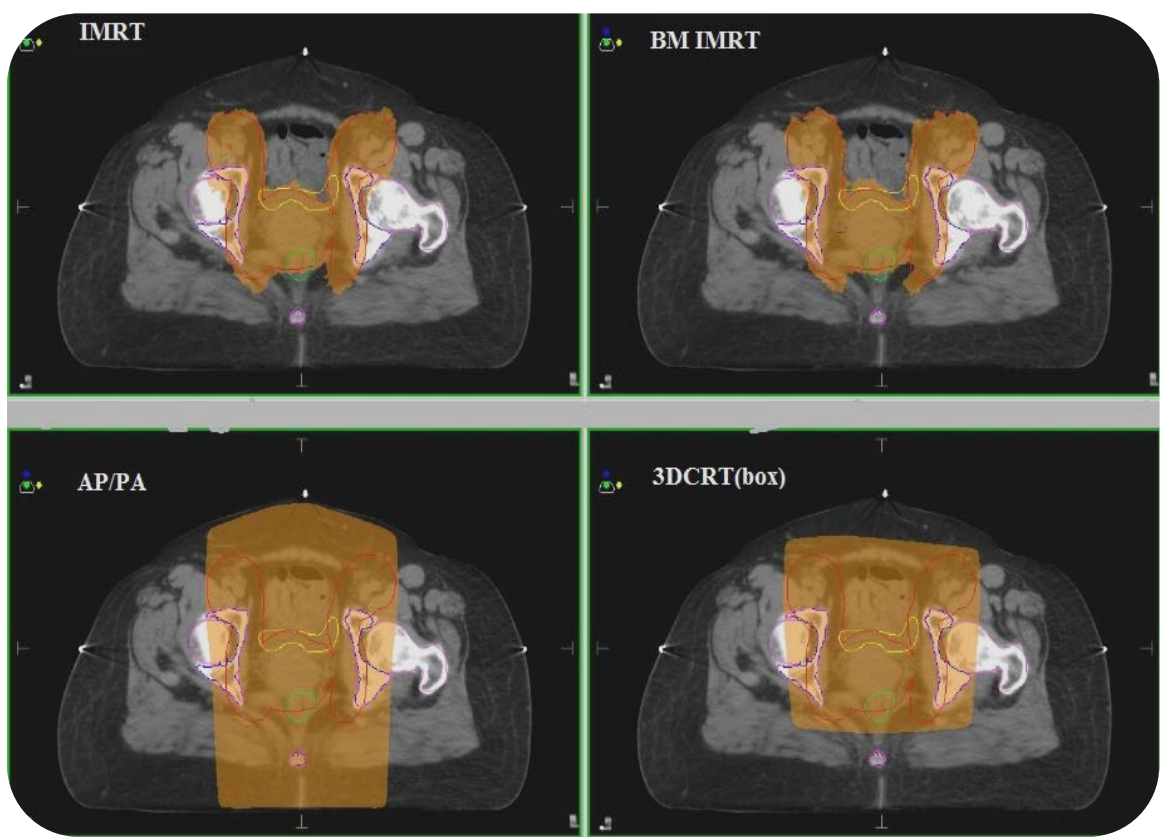

Figure 1. Colour-wash (95\%) showing dose distributions obtained for IMRT, BMSIMRT, AP/PA and 3DCRT techniques. PTV (red), BM (pink) bladder (yellow), and rectum (green). 


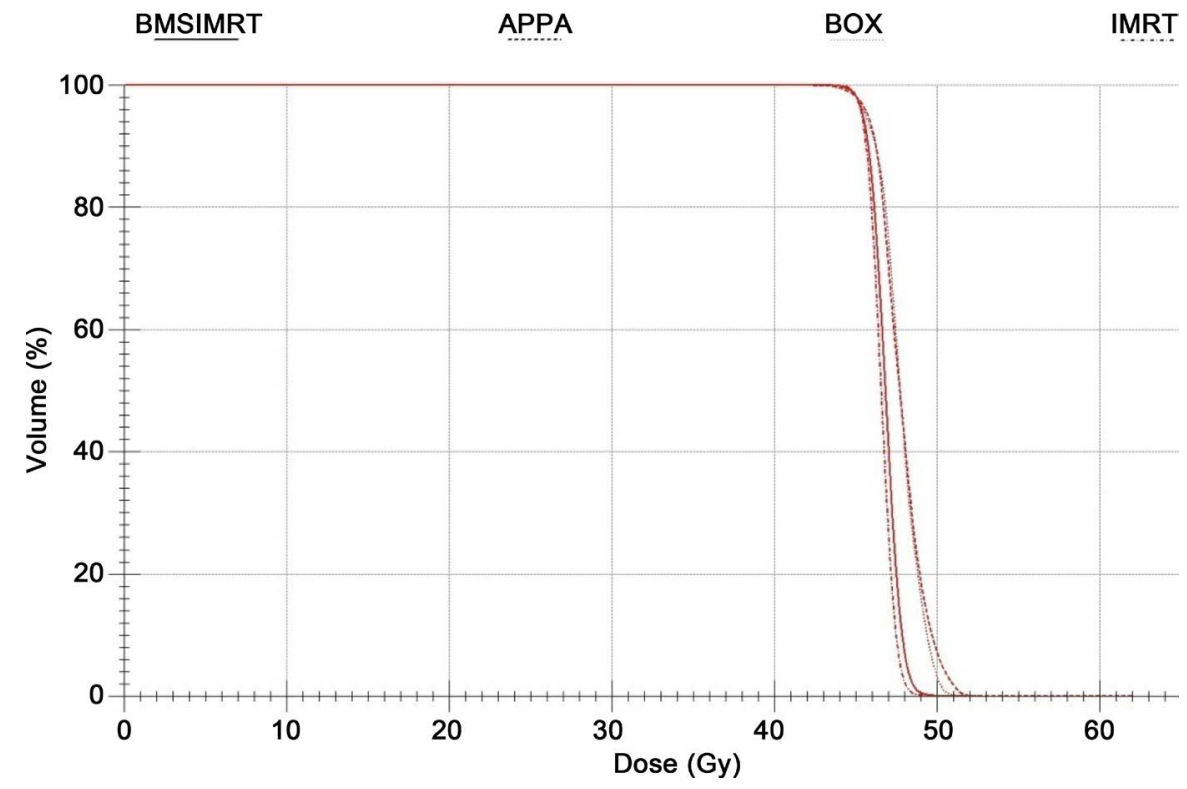

Figure 2. DVH for PTV dose distribution comparing BMS-IMRT, AP/PA, 3DCRT and IMRT.

3DCRT technique. The AP/PA plans produced sharp fall-off in isodose lines, 3DCRT plans showed better dose coverage to the PTV than AP/PA techniques, however 3DCRT plans showed that there is a greater dose to the PBM.

Figure 2, showed DVH for PTV dose distribution comparing BMS-IMRT, AP/PA, 3DCRT and IMRT. Both BMS-IMRT and IMRT plans showed better dose coverage to the PTV and reduced the dose to organs compared to 3DCRT and AP/PA. BMS-IMRT plans limited total dose distribution to the PTV in the regions where the $\mathrm{BM}$ is close to the PTV compared to IMRT, 3DCRT and AP/PA.

BMS-IMRT reduced the BM volume receiving dose 20, 30, 40 and $45 \mathrm{~Gy}$ compared with 3DCRT and IMRT plans. A significant reduction in V20 BMSIMRT compared with 3DCRT $(p<0.03)$. The PBM volume receiving 5, 10 and 20 Gy with AP/PA was lower than BMS-IMRT ( $p<0.01, p<0.001$ and $p<0.04$ respectively). There was a reduction in the volume of BMS irradiated (V30, V40 and V45) with $\mathrm{AP} / \mathrm{PA}$ techniques, but the differences were not statistically significant ( $p<0.33, p<0.08$ and $p<0.18$ respectively).

There is a significant reduction in BMS-IMRT included iliac bone marrow V10 and V20 compared with AP/PA $(p<0.03)$.

Table 1 shows comparison of volumes of the pelvic bone marrow (include: iliac, pubis Lumbosacral), rectum and bladder that received 5, 10, 20, 30, 40 and $45 \mathrm{~Gy}$ for four techniques.

Figure 3 shown DVH comparing the BMS-IMRT, AP/PA, 3DCRT, and IMRT treatment plans, where there was significant dose reduction in the BMS-IMRT technique than other techniques.

Also, BMS-IMRT provided a significant reduction in the rectum and bladder volumes receiving 40 and $45 \mathrm{~Gy}$ compared with $\mathrm{AP} / \mathrm{PA}$ and 3DCRT planning techniques as shown in Figure 4 and Figure 5. 
Table 1. (a)-(c). Organs at risk dose-volume comparison.

(a)

\begin{tabular}{|c|c|c|c|c|c|c|}
\hline \multirow{2}{*}{\multicolumn{2}{|c|}{$\begin{array}{c}\text { Organ at Risk } \\
\text { Volume Received } \times \text { Gy }\end{array}$}} & \multicolumn{2}{|c|}{ Ant/Post } & \multicolumn{2}{|c|}{ BMS-IMRT } & \multirow[b]{2}{*}{$P$-value } \\
\hline & & $\begin{array}{c}\text { Mean } \\
(\%)\end{array}$ & $\begin{array}{l}\text { Median } \\
\text { (Range) }\end{array}$ & $\begin{array}{c}\text { Mean } \\
(\%)\end{array}$ & $\begin{array}{l}\text { Median } \\
\text { (Range) }\end{array}$ & \\
\hline \multirow{6}{*}{ PBM (all) } & V5 & 77.17 & $77.64(72-82)$ & 98.96 & $98.47(98-100)$ & 0.01 \\
\hline & V10 & 72.05 & $73.90(65-77)$ & 94.49 & $95.05(89-99)$ & 0.001 \\
\hline & V20 & 68.09 & $70.62(61-73)$ & 78.03 & $78.33(73-83)$ & 0.04 \\
\hline & V30 & 63.72 & $66.19(57-68)$ & 58.38 & $59.79(55-60)$ & 0.33 \\
\hline & V40 & 59.16 & $62.05(53-63)$ & 37.68 & $34.58(34-45)$ & 0.08 \\
\hline & V45 & 70.64 & $57.60(54-100)$ & 46.58 & $20.27(19-100)$ & 0.18 \\
\hline \multirow{6}{*}{ Iliac } & V5 & 71.94 & $69.28(69-77)$ & 76.02 & $99.55(5-100)$ & 0.01 \\
\hline & V10 & 65.30 & $63.38(60-73)$ & 75.75 & $96.68(10-100)$ & 0.03 \\
\hline & V20 & 59.77 & $58.46(52-69)$ & 66.7 & $79.09(20-89)$ & 0.03 \\
\hline & V30 & 55.71 & $53.21(45-66)$ & 51.57 & $51.62(30-73)$ & 0.28 \\
\hline & V40 & 48.37 & $46(38-61)$ & 35.42 & $34.93(20-51)$ & 0.12 \\
\hline & V45 & 33.71 & $36.73(23-42)$ & 25.19 & $22.87(10-45)$ & 0.12 \\
\hline \multirow{6}{*}{ Pubis } & V5 & 92.18 & $93.87(83-100)$ & 98.05 & $100(100-100)$ & 0.27 \\
\hline & V10 & 86.84 & $88.67(72-100)$ & 93.62 & $100(100-100)$ & 0.29 \\
\hline & V20 & 83.36 & $84.05(66-100)$ & 88.28 & $86.16(85-98)$ & 0.42 \\
\hline & V30 & 77.73 & $74.88(61-97)$ & 57.02 & $61.74(61-73)$ & 0.21 \\
\hline & V40 & 73.04 & $70.33(54-95)$ & 47.28 & $41.56(36-98)$ & 0.44 \\
\hline & V45 & 63.20 & $66.90(31-92)$ & 41.87 & $24.32(22-28)$ & 0.14 \\
\hline \multirow{6}{*}{ Lumbosacral } & V5 & 75.49 & $98.48(5-100)$ & 75.97 & $99.44(5-100)$ & 0.42 \\
\hline & V10 & 74.82 & $94.64(10-100)$ & 74.42 & $93.84(10-100)$ & 0.4 \\
\hline & V20 & 76.38 & $92.75(20-100)$ & 75.14 & $90.28(20-100)$ & 0.2 \\
\hline & V30 & 78.16 & $91.31(30-100)$ & 68.59 & $77.16(30-90)$ & 0.2 \\
\hline & V40 & 79.44 & $88.89(40-100)$ & 58.20 & $57.47(40-78)$ & 0.16 \\
\hline & V45 & 78.43 & $84.43(45-100)$ & 47.31 & $43.98(32-70)$ & 0.13 \\
\hline \multirow{3}{*}{ Rectum } & V30 & 100 & $100(100-100)$ & 97.15 & $98.13(93-100)$ & 0.27 \\
\hline & V40 & 100 & $100(100-100)$ & 73.90 & $71.33(71-80)$ & 0.011 \\
\hline & V45 & 100 & $100(100-100)$ & 45.62 & $48.98(35-55)$ & 0.01 \\
\hline \multirow{3}{*}{ Bladder } & V30 & 100 & $100(100-100)$ & 92.89 & $91.91(90-97)$ & 0.08 \\
\hline & V40 & 100 & $100(100-100)$ & 70.88 & $68.99(64-80)$ & 0.027 \\
\hline & V45 & 99.97 & $100(100-100)$ & 49.35 & $50.96(39-58)$ & 0.011 \\
\hline
\end{tabular}


(b)

\begin{tabular}{|c|c|c|c|c|c|c|}
\hline \multirow{2}{*}{\multicolumn{2}{|c|}{$\begin{array}{c}\text { Organ at Risk } \\
\text { Volume Received } \times \text { Gy }\end{array}$}} & \multicolumn{2}{|c|}{ 3DCRT (Box technique) } & \multicolumn{2}{|c|}{ BMS-IMRT } & \multirow[b]{2}{*}{$P$-value } \\
\hline & & Mean & $\begin{array}{l}\text { Median } \\
\text { (Range) }\end{array}$ & Mean & $\begin{array}{l}\text { Median } \\
\text { (Range) }\end{array}$ & \\
\hline \multirow{6}{*}{ PBM (all) } & V5 & 99.05 & $99.16(98-100)$ & 98.96 & $98.47(98-100)$ & 0.9 \\
\hline & V10 & 95.66 & $95.64(95-97)$ & 94.49 & $95.05(89-99)$ & 0.6 \\
\hline & V20 & 91.81 & $91.99(91-92)$ & 78.03 & $78.33(73-83)$ & 0.03 \\
\hline & V30 & 65.16 & $67.73(57-70)$ & 58.38 & $59.79(55-60)$ & 0.3 \\
\hline & $\mathrm{V} 40$ & 52.25 & $51.09(50-55)$ & 37.68 & $34.58(34-45)$ & 0.08 \\
\hline & V45 & 63.68 & $64.08(44.02-100)$ & 46.58 & $20.27(19-100)$ & 0.18 \\
\hline \multirow{6}{*}{ Iliac } & V5 & 98.05 & $99.63(95-100)$ & 76.02 & $99.55(5-100)$ & 0.44 \\
\hline & V10 & 93.62 & $95.14(88-97)$ & 75.75 & $96.68(10-100)$ & 0.37 \\
\hline & V20 & 88.28 & $91.29(81-92)$ & 66.7 & $79.09(20-89)$ & 0.06 \\
\hline & V30 & 57.02 & $51.83(49-70)$ & 51.57 & $51.62(30-73)$ & 0.22 \\
\hline & V40 & 47.28 & $41.05(37-64)$ & 35.42 & $34.93(20-51)$ & 0.01 \\
\hline & V45 & 41.87 & $35.08(32-59)$ & 25.19 & $22.87(10-45)$ & 0.01 \\
\hline \multirow{6}{*}{ Pubis } & V5 & 99.85 & $100(100-100)$ & 98.05 & $100(100-100)$ & 0.4 \\
\hline & V10 & 98.42 & $99.57(96-100)$ & 93.62 & $100(100-100)$ & 0.36 \\
\hline & V20 & 94.90 & $96.91(88-100)$ & 88.28 & $86.16(85-98)$ & 0.24 \\
\hline & V30 & 78.82 & $80.65(56-100)$ & 57.02 & $61.74(61-73)$ & 0.28 \\
\hline & V40 & 67.83 & $71.83(35-97)$ & 47.28 & $41.56(36-98)$ & 0.46 \\
\hline & V45 & 59.2 & $57.36(28-92)$ & 41.87 & $24.32(22-28)$ & 0.17 \\
\hline \multirow{6}{*}{ Lumbosacral } & V5 & 75.57 & $98.63(5-100)$ & 75.97 & $99.44(5-100)$ & 0.4 \\
\hline & V10 & 74.98 & $94.96(10-100)$ & 74.42 & $93.84(10-100)$ & 0.4 \\
\hline & $\mathrm{V} 20$ & 76.39 & $92.79(20-100)$ & 75.14 & $90.28(20-100)$ & 0.18 \\
\hline & V30 & 78.82 & $88.67(30-98)$ & 68.59 & $77.16(30-90)$ & 0.18 \\
\hline & V40 & 62.69 & $64.68(40-81)$ & 58.20 & $57.47(40-78)$ & 0.22 \\
\hline & V45 & 56.53 & $52.26(45-77)$ & 47.31 & $43.98(32-70)$ & 0.04 \\
\hline \multirow{3}{*}{ Rectum } & V30 & 100 & $100(100-100)$ & 97.15 & $98.13(93-100)$ & 0.27 \\
\hline & $\mathrm{V} 40$ & 94.65 & $99.9(84-100)$ & 73.90 & $71.33(71-80)$ & 0.04 \\
\hline & V45 & 86.9 & $88.90(75-98)$ & 45.62 & $48.98(35-55)$ & 0.03 \\
\hline \multirow{3}{*}{ Bladder } & V30 & 99.97 & $100(95-100)$ & 92.89 & $91.91(90-97)$ & 0.08 \\
\hline & $\mathrm{V} 40$ & 98.35 & $100(87-100)$ & 70.88 & $68.99(64-80)$ & 0.027 \\
\hline & V45 & 95.63 & $100(100-100)$ & 49.35 & $50.96(39-58)$ & 0.01 \\
\hline
\end{tabular}


(c)

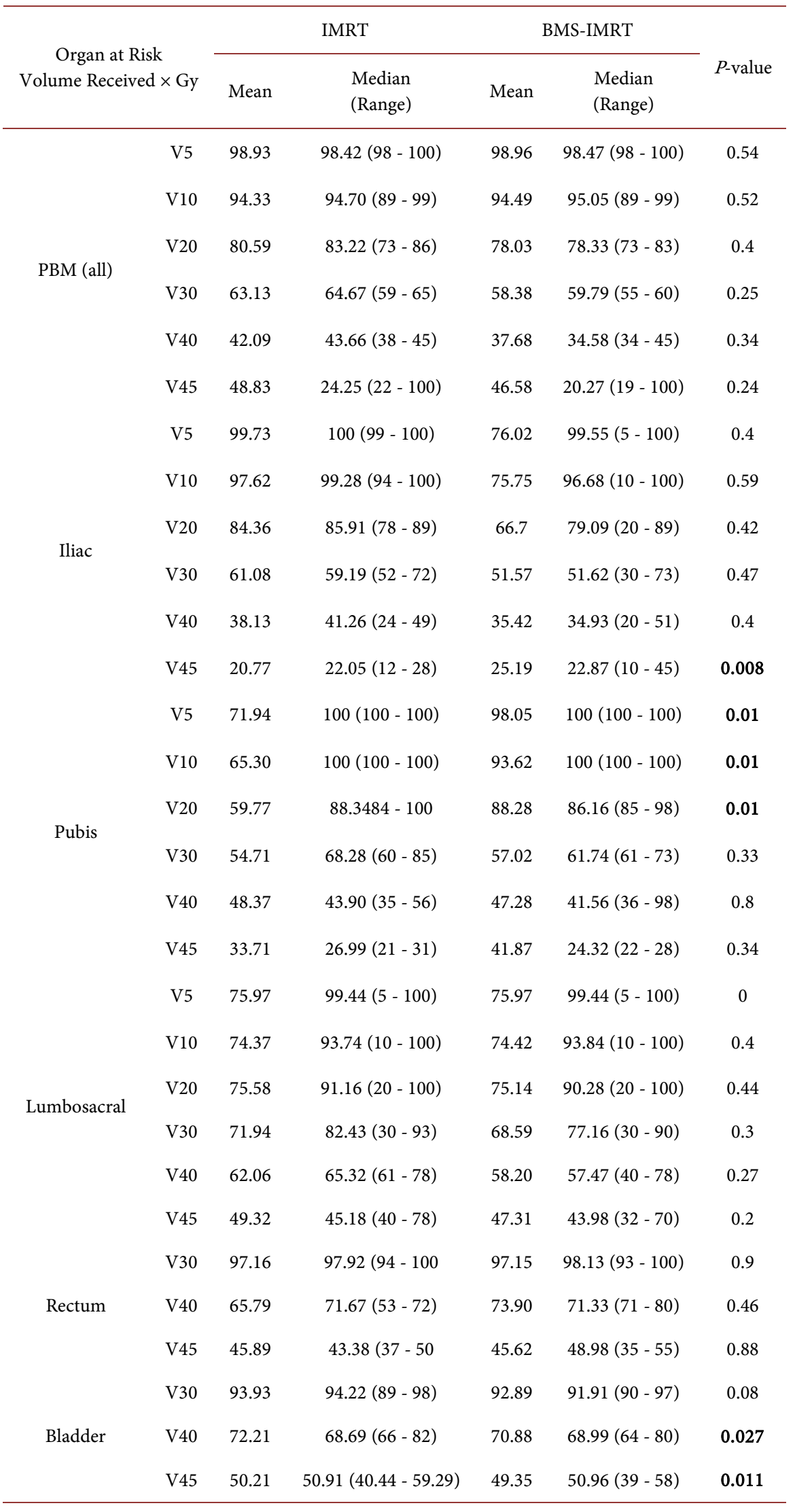




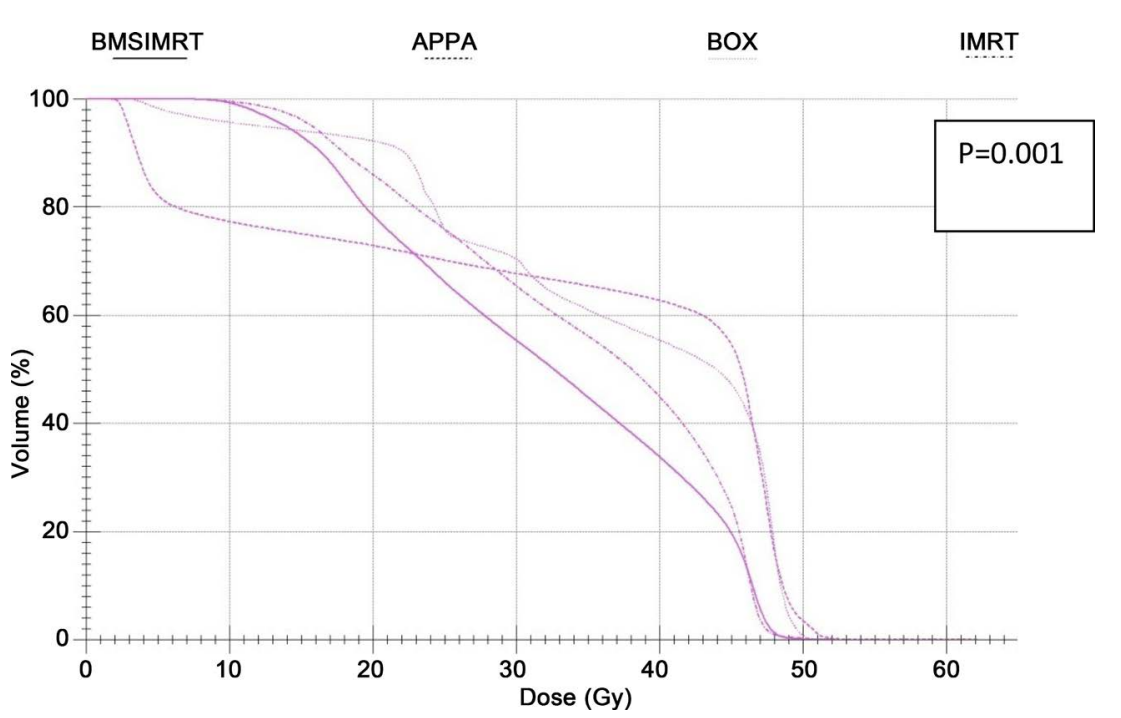

Figure 3. PBM DVH dose distribution comparing BMS-IMRT, AP/PA, 3DCRT and IMRT.

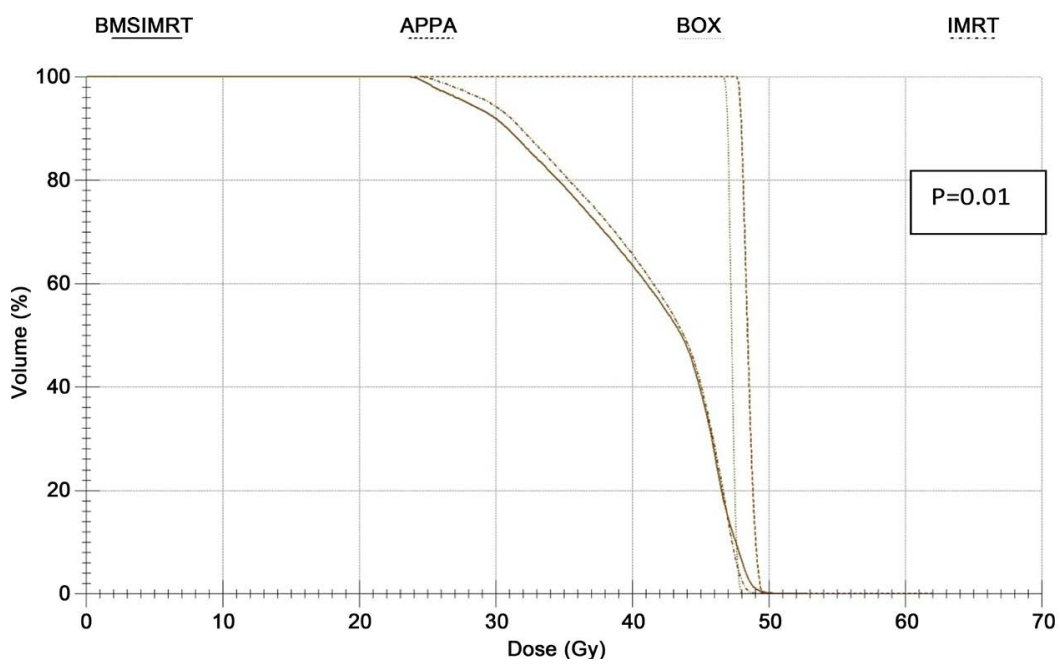

Figure 4. Bladder dose distribution comparing BMS-IMRT, AP/PA, 3DCRT and IMRT.

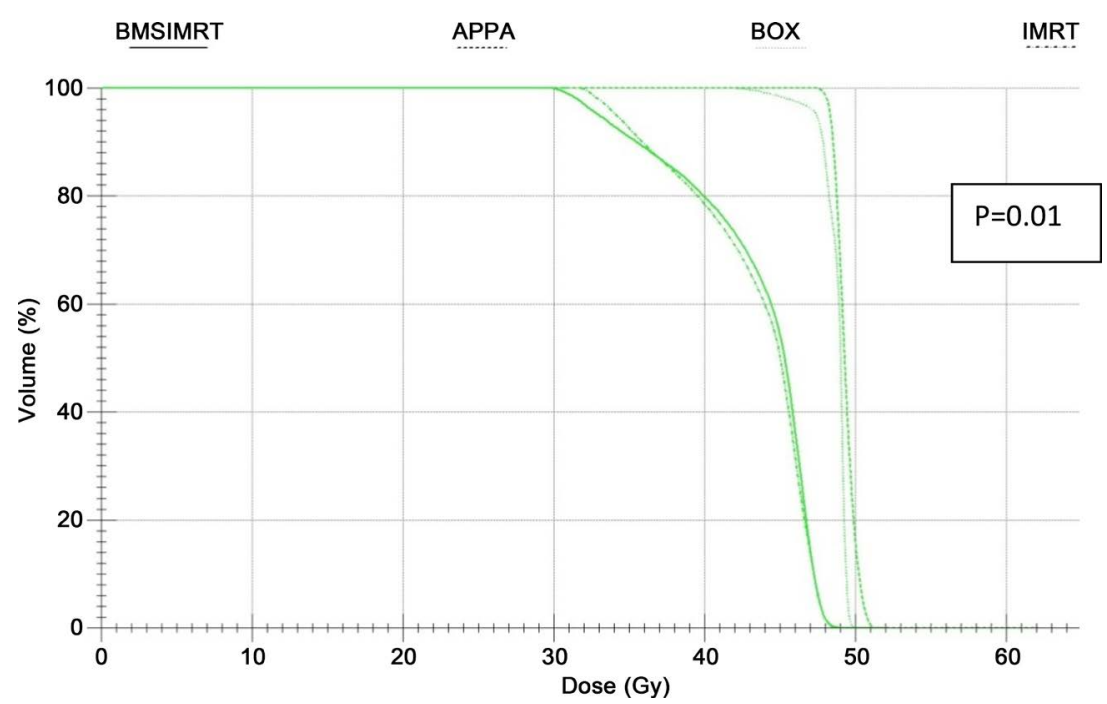

Figure 5. Rectum dose distribution comparing BMS-IMRT, AP/PA, 3DCRT and IMRT. 


\section{Discussion}

Great concern has been raised for the use of IMRT in patients with gynecologic malignancies. IMRT reduced the volume of small bowel, bladder, and rectum irradiated in cases with cervical and endometrial cancers in comparison to conventional whole pelvic radiotherapy [8] [9] [10] [11].

In the present study, BMS-IMRT was superior in reducing the BM volume (V20, V30, V40, V45) compared with 3DCRT and IMRT.

The V5, V10 and V20 of the PBM were lower with AP/PA than BMS-IMRT ( $p$ $<0.01, p<0.001$ and $p<0.04$ respectively).

Brixey et al. [12] reported that hematological toxicity was minimized by using IMRT plan compared to four-field box. The dose to Iliac, lumbar, and sacral BM were also reduced with IMRT, although the aim was not planned to spare the BM.

Lujan et al 2003 [13], reported that BMS IM-WPRT treatment plans demonstrated a significant reduction of the volume of BM receiving $>40 \%$ (18 Gy) of the prescription dose ( $45 \mathrm{~Gy}$ ) compared to both IM-WPRT and four-field treatment.

Wong et al 2005 [14], used an intensity-modulated arc therapy (IMAT) for treating patients with endometrial cancer. Results showed that using two anterior intensity-modulated arcs produced good cover for the PTVs. Also; IMAT technique allowed sparing of the iliac similar to 8-field IMRT. But the 8-field IMRT resulted in better dose uniformity than IMAT in the target volumes coverage.

Regarding postoperative cervical cancer patients, Chen et al. [15], demonstrated that using IMRT reduced PBM irradiation compared with four-field box techniques.

Ahmed et al 2004 [16], studied the feasibility of dose escalation to para-aortic lymph node (PALN) through using intensity modulated radiation therapy (IMRT) with reducing the dose to bone marrow, bowel, spinal cord, and kidneys in comparison to conventional radiation to PALNs in patients with locally advanced cervical cancer and PALN metastases. The study showed that IMRT significantly reduced the volume of bone marrow receiving a dose $40 \mathrm{~Gy}$ compared to the AP/PA and 4-field box techniques with a median of $21.3 \%, 98 \%$, and $49.7 \%$, respectively.

Mell et al 2008 [17], compared BMS-IMRT with four-field box and AP/PA techniques in the treatment of cancer cervix. BMS-IMRT was better than the four-field box technique in reducing the dose to the PBM, small bowel, rectum, and bladder. Also the PBM volume receiving a dose $>16.4$ Gy was less in the BMS-IMRT plans compared to AP-PA plans.

\section{References}

[1] Eifel, P.J., Winter, K., Morris, M., Levenback, C., Grigsby, P.W., Cooper, J., Rotman, M., Gershenson, D. and Mutch, D.G. (2004) Pelvic Irradiation with Concurrent 
Chemotherapy versus Pelvic and Para-Aortic Irradiation for High-Risk Cervical Cancer: An Update of Radiation Therapy Oncology Group Trial (RTOG) 90-01. Journal of Clinical Oncology, 22, 872-880. https://doi.org/10.1200/JCO.2004.07.197

[2] Rose, P.G., Ali, S., Watkins, E., Thigpen, J.T., Deppe, G., Clarke-Pearson, D.L. and Insalaco, S. (2007) Gynecologic Oncology Group. Long-Term Follow-Up of a Randomized Trial Comparing Concurrent Single Agent Cisplatin, Cisplatin-Based Combination Chemotherapy, or Hydroxyurea During Pelvic Irradiation for Locally Advanced Cervical Cancer: A Gynecologic Oncology Group Study. Journal of Clinical Oncology, 25, 2804-2810. https://doi.org/10.1200/JCO.2006.09.4532

[3] Green, J.A., Kirwan, J.M., Tierney, J.F., et al. (2001) Survival and Recurrence after Concomitant Chemotherapy and Radiotherapy for Cancer of the Uterine Cervix: A Systematic Review and Meta-Analysis. Lancet, 358, 781-786.

https://doi.org/10.1016/S0140-6736(01)05965-7

[4] Mell, L.K., Kochanski, J.D., Roeske, J.C., et al. (2006) Dosimetric Predictors of Acute Hematologic Toxicity in Cervical Cancer Patients Treated with Concurrent Cisplatin and Intensity-Modulated Pelvic Radiotherapy. International Journal of Radiation Oncology, Biology, Physics, 66, 1356-1365.

https://doi.org/10.1016/j.ijrobp.2006.03.018

[5] Mauch, P., Constine, L., Greenberger, J., et al. (1995) Hematopoietic stem cell compartment: acute and late effects of radiation therapy and chemotherapy. International Journal of Radiation Oncology, Biology, Physics, 31, 1319-1339. https://doi.org/10.1016/0360-3016(94)00430-S

[6] Mell, L.K., Schomas, D.A., Salama, J.K., et al. (2008) Association between Bone Marrow Dosimetric Parameters and Acute Hematologic Toxicity in Anal Cancer Patients Treated with Concurrent Chemotherapy and Intensity Modulated Radiotherapy. International Journal of Radiation Oncology, Biology, Physics, 70, 1431-1437. https://doi.org/10.1016/j.ijrobp.2007.08.074

[7] Rose, B.S., Aydogan, B., Liang, Y., et al. (2011) Normal Tissue Complication Probability Modeling of Acute Hematologic Toxicity in Cervical Cancer Patients Treated with Chemoradiotherapy. International Journal of Radiation Oncology, Biology, Physics, 79, 800-807. https://doi.org/10.1016/j.ijrobp.2009.11.010

[8] Roeske, J.C., Lujan, A.E., Rotmensch, J., et al. (2000) Intensity Modulated Whole Pelvic Radiation Therapy in Patients with Gynecological Malignancies. International Journal of Radiation Oncology, Biology, Physics, 48, 1613-1621. https://doi.org/10.1016/S0360-3016(00)00771-9

[9] Mundt, A.J., Roeske, J.C. and Lujan, A.E. (2002) Intensity Modulated Radiation Therapy in Gynecologic Malignancies. Medical Dosimetry, 27, 131-136. https://doi.org/10.1016/S0958-3947(02)00095-X

[10] Chen, Q., Izadifar, N., King, S., et al. (2001) Comparison of IMRT with 3-D CRT for Gynecologic Malignancies. International Journal of Radiation Oncology, Biology, Physics, 51, 332.

[11] Selvaraj, R.N., Rerszten, K., King, G.C., et al. (2001) Conventional 3-D versus Intensity Modulated Radiotherapy for the Adjuvant Treatment of Gynecologic Malignancies: A Comparative Study of Dose-Volume Histograms and the Potential Impact on Toxicities. International Journal of Radiation Oncology, Biology, Physics, $51,218$.

[12] Brixey, C.J., Roeske, J.C., Lujan, A., et al. (2002) Impact of Intensity-Modulated Radiotherapy on Acute Hematologic Toxicity in Women with Gynecologic Malignancies. International Journal of Radiation Oncology, Biology, Physics, 54, 1388-1396. 
[13] Lujan, A., Mundt, A.J., Yamada, D., et al. (2003) Intensity-Modulated Radiotherapy as a Means of Reducing Dose to Bone Marrow in Gynecologic Patients Receiving Whole Pelvic Radiotherapy. International Journal of Radiation Oncology, Biology, Physics, 57, 516-521.

[14] Wong, E., D’Souza, D.P., Chen, J.Z., et al. (2005) Intensity-Modulated Arc Therapy for Treatment of High-Risk Endometrial Malignancies. International Journal of Radiation Oncology, Biology, Physics, 61, 830-841.

[15] Chen, M.F., Tseng, C.J., Tseng, C.C., et al. (2007) Clinical Outcome in Posthysterectomy Cervical Cancer Patients Treated with Concurrent Cisplatin and Intensity-Modulated Pelvic Radiotherapy: Comparison with Conventional Radiotherapy. International Journal of Radiation Oncology, Biology, Physics, 67, 1438-1444.

[16] Ahmed, R.S., Kim, R.Y., Duan, J., et al. (2004) IMRT Dose Escalation for Positive Para-Aortic Lymph Nodes in Patients with Locally Advanced Cervical Cancer While Reducing Dose to Bone Marrow and Other Organs at Risk. International Journal of Radiation Oncology, Biology, Physics, 60, 505-512.

[17] Mell, L.K., Tiryaki, H., Ahn, K.H., et al. (2008) Dosimetric Comparison of Bone Marrow-Sparing IMRT versus Conventional Techniques for Treatment of Cervical Cancer. International Journal of Radiation Oncology, Biology, Physics, 71, 1504-1510. 